\title{
Introduction to Architectural Structures: Lessons Learned from Parti Pris Pedagogy
}

\section{Prof. Keith E. Hedges, Drury University}

Keith Hedges is an Assistant Professor of Architecture and teaches the architectural structures sequence at Drury University. Keith's teaching repertoire includes seventeen different courses of engineering topics at NAAB (architecture) and architecture topics at ABET (engineering) accredited institutions. His interests involve the disciplinary knowledge gap between architecture and engineering students in higher education. 


\title{
Introduction to architectural structures: Lessons learned from parti pris pedagogy
}

\begin{abstract}
$\underline{\text { Abstract }}$
The architectural academy recognizes mathematics and its distance from the design process as the primary challenges confronting structures educators in the liberal arts environment. Although architecture and engineering educators have pedagogical polarity, the germaneness of mathematics is not the fundamental problem. The psychology of how we learn has changed. Educators frequently model structures with a bottom-up approach through the gradual accrual of prerequisite knowledge, which follows the older associationist-behaviorist paradigm. In the newer cognitive paradigm, scientific psychologists recognize that the mental framework, or schemata of the learner, supersedes the prerequisite knowledge. The cognitive paradigm suggests that explicit information precedes the discovery of implicit knowledge in a top-down approach, in harmony with the life experiences inside the architecture studio culture. The studio project engages the parti pris process that begins with a central big idea prior to its refinement. A need exists to explore a pedagogy that commences with the central idea of architectural structures. This paper outlines a new approach for a structures pedagogy that reverses the content sequence to parallel the architecture student schemata in an introductory architectural structures course. A quantitative analysis of unobtrusive data describes thirty-one second-year architecture students studying in either a traditional associationist-behaviorist course content sequence or a reverse content parti pris cognitive sequence at an institute of higher education rooted in the liberal arts. The findings indicate that the parti pris pedagogy improves student performance in nongraphical multiple-choice examinations. The educational lessons learned offer reflections in the areas of instruction, content, and student outcomes.

$\underline{\text { Introduction }}$
\end{abstract}

"The only thing that interferes with my learning is my education." - Albert Einstein.

Society thinks of education as a gateway to success, but sometimes education does not reach all learners or even some Nobel Prize winners in the same manner. Beeston noted the challenges that Einstein overcame with his difficulty of the common mechanical, or rote learning of the time.$^{[1]}$ The quote is a reminder that not all students learn equally and sometimes pedagogy should adapt when students cannot.

Many architects believe that mathematics interferes with design learning, while engineers understand and advocate its eloquence. The architectural academy cites dissatisfaction with structures pedagogy through its detachment with the design process and its emphasis on mathematics. ${ }^{[2,3,4]}$ Engineers commonly embrace mathematics in architecture. Mario Salvadori thought that "to satisfy the prerequisites for an understanding of structures you have to abolish certain prejudices against mathematics." ${ }^{[5]}$ Even though mathematics has dominated the discourse, I posit that understanding how students learn is more important than debating whether mathematics should be the prevailing underpinnings of architectural structures. 
Since Salvadori, the psychology of how we learn transitioned from an associationist-behaviorist (A-B) paradigm to a cognitive paradigm, without a comparable shift in architectural structures education. Scientific psychologists recognize that the sequential accrual of prerequisite knowledge fits in the older A-B paradigm. ${ }^{[6]}$ Researchers show that the current cognitive paradigm places an emphasis on the schemata of the learners. The mental framework of the architectural education experience supersedes the prerequisite knowledge. The architecture students learn and retrieve information differently while possessing the same prerequisite knowledge as the engineering students because of the disparities in their mental frameworks. Structures education is commonly a linear progression from mathematics, physics, rigid body statics, mechanics of deformable bodies, structural materials design and analysis, to lateral forces and overall building behavior. ${ }^{[7]}$ The gradual accrual of prerequisite knowledge is suited for the engineering schemata rather than the architecture schemata. A need exists to develop new way of thinking about this current problem and explore a structures pedagogy that is consistent with the mental framework of architecture students.

In response to that need, an introductory architectural structures course was available to describe, "How does the parti pris reverse content sequence differ from the traditional sequence in terms of student performance in architectural structures at a private liberal arts institution?" A quantitative study in the group comparison tradition examines the question. The purpose is to investigate the schemata notion by comparing the achievements of a parti pris group and a traditional group. This paper describes the development of a recurring parti pris course and a subsequent comparison to conventional methods.

\section{Basis for Pedagogical Change}

Change requires understanding the structures content in architecture programs, the discourse between architecture and engineering pedagogy, and the foundations of scientific psychology.

\section{Defining Architectural Structures}

The architectural accreditation board is the only governing body that enforces an education standard for the minimum student performance in architectural structures. The National Architectural Accreditation Board, Inc. (NAAB) requires that programs demonstrate satisfactory evidence of student achievement within a discrete structural systems criterion and under a broader design studio situation. NAAB defines structural systems as the "understanding of the basic principles of structural behavior in withstanding gravity and lateral forces and the evolution, range, and appropriate application of contemporary structural systems." ${ }^{\text {"[8] }}$ The integrated architectural solutions realm (comprehensive design) implies a closer engagement between technology and the design studio through the new integrative design criterion that reads, "Ability to produce an architectural solution that demonstrates the ability to make design decisions about a single project while demonstrating broad integration and consideration of environmental stewardship, technical documentation, accessibility, site conditions, life safety, environmental systems, structural systems, and building envelope systems and assemblies." ${ }^{[8]}$ Since NAAB does not mandate a specific pedagogy or number of credit hours, the structural systems criterion is a broad condition with a significant breadth of academic freedom. 
The architectural licensing board and the academy have no curricular enforcement powers, although they do approximate and interpret structures as NAAB constituents. The National Council of Architectural Registration Boards, Inc. (NCARB) defines structural systems as, "The study of the basic structural elements of buildings, their interaction as a support system, the forces that act on and in buildings, and the principles, theory, and appropriate applications of these systems." ${ }^{[9]}$ The NCARB approximates that the academy, on average, assigns six credithours to structures exclusive of any prerequisite mathematics and physics coursework. The American Collegiate Schools of Architecture (ACSA) is the most descriptive in their interpretation of structures in accredited programs. The ACSA describes a framework of content and sequence, "Beginning with algebra, trigonometry, and physics, most schools require at least one course in calculus and descriptive geometry before the introduction of engineering statics (a development of the study of vector forces, from elementary physics). There may be an additional course on structural materials, particularly in engineering-oriented programs. The actual structures courses may proceed in various sequences by reference to the type of structural element (beam, column, etc.) or the structural material itself (wood, steel, reinforced concrete)," while extricating and distancing the big idea of lateral forces, "The problem of designing a structure to withstand the forces of gravity, wind, and earthquakes is usually addressed through another series of courses." ${ }^{[7]}$

\section{The History of Discourse}

The pedagogical approaches shifted from the Beaux Arts to the Bauhaus tradition between 1925 and 1950 thereby inducing an ongoing turmoil in architecture education. Henry Kamphoefner described the Beaux Arts education as having a focus on the rendered drawing of the façade with the students being informed to "ignore the structure," as this would be accomplished by others. ${ }^{[10]} \mathrm{He}$ continued that structures pedagogy began a transformation as Walter Gropius came to Harvard University, "The Schools have been re-thinking their programs to bring mathematics, mechanics and the science of structure into sharper focus and into a clearer relationship with the design of space." $[10]$ The architecture students contributed to the American movement as they pushed back against the Beaux Arts traditions in favor of more modern approaches. ${ }^{[1]}$ The education that supports modern architecture elevated mathematics in the structures curriculum, but also brought forth enduring pedagogical discourse.

The ACSA recognizes the disparity between the architectural design and the structural design processes, and the mathematical priority as the two fundamental problems with structures pedagogy. In 1976, the ACSA formed an ad hoc committee based on their frustrations of structures pedagogy. In a memo from member Richard Bender, they commented on the structures sequence, "However, divorced as it usually is from involvement with the total process of design, this sequence has resulted in architectural graduates who have no understanding of the basic principles involved, cannot apply them, nor retain for a significant period after graduation the basic core of material encountered." "[4] The committee was concerned that structures pedagogy evolved from engineering programs without a connection to the total process of design. In 1994, David Faoro surveyed the ACSA structures faculty regarding pedagogy. The survey found most teachers were civil engineers who recognized a deficit in connecting the structures and studio courses, "The teachers who responded placed their highest priority on mathematical analysis of structural members, and on qualitative/ intuitive understanding of 
structural systems. The respondents gave a much lower priority to understanding the implications of structural systems in the design process. Only 14\% felt that their technical courses were well integrated with design studios." "[5] The ACSA indicates a diminishing representation in the mastery of structural calculations, "Today, most final structural calculations are done by engineering specialists. A mastery of structural calculations is not essential for the architect, but an understanding of structural principles and material properties is." "[7]

A point counterpoint from the architectural and engineering perspectives addresses the pedagogical disparity. "We spend far too much time teaching calculations, which are the least important thing about structural design. It's much more important that students learn real-world structural concepts and develop structural intuition ... The mathematical underpinnings of structural design are wonderfully elegant and clear. They give us an ideal way of unraveling the mysteries of structural behavior and developing in students an intuitive sense of how structures work." ${ }^{[2]}$ Figure 1 shows the full point counterpoint that articulates the opposing notions. The point counterpoint is evidence that a divide remains years after the 1976 ACSA Memo regarding the appropriate structures pedagogy. Ironically, the terminal words of "fuzzy conceptualizations" (Figure 1(b)) align with the notion of the parti.

We spend far too much time teaching calculations, which are the least important thing about structural design. It's much more important that students learn real-world structural concepts and develop structural intuition. We should spend much more time teaching students to select an appropriate structural material and system for any building, lay out the system in a way that works well with the building's form and space, and assign approximate sizes to the structural members. The exact sizing of the members will be done mostly by engineers and computers in the real world, so architecture students need be taught only a basic repertoire of calculations.

(a)
The mathematical underpinnings of structural design are wonderfully elegant and clear. They give us an ideal way of unraveling the mysteries of structural behavior and developing in students an intuitive sense of how structures work. They lead naturally to consideration of detailed design calculations in all structural materials. This prepares students to perform well on the architectural registration exam. It also prepares them to communicate with structural engineers in their own language. The teaching of a field so precise as structures should not be diluted with rough approximations and fuzzy conceptualizations.

Figure 1. The polarities of teaching structures: (a) architecture; and (b) engineering. ${ }^{[2]}$

\section{The Architecture Student Schemata}

Scientific psychology recognized a swing from an associationist-behaviorist paradigm (prerequisite knowledge basis) towards the cognitive paradigm (mental framework basis) a few decades ago. The role of knowledge in the A-B approach is "knowledge acquisition as prerequisite knowledge use in problem solving," and the organization of content in the A-B approach is to "make the organization explicit." $[6]$ The linear progression of structural courses and topics is consistent with A-B, which builds on the accrual of prerequisite knowledge from the bottom-up. In the cognitive approach, the prerequisite knowledge is subordinate to the knowledge associated with a student's particular mental framework. The role of knowledge is "knowledge as basis for reasoning, decision making," and the organization of content for the cognitive approach which is to "help discovery of implicit organization." "[6] Anderson and Pichert found that the knowledge retrieval is independent from the encoding process. ${ }^{[12]}$ Therefore, the cognitive paradigm suggests that explicit information consciously precedes then one discovers 
the implicit knowledge unconsciously, which is a top-down perspective. The student learner will retrieve knowledge from their mental framework based on their contextualized life experiences or their schemata. The architectural design process is consistent with the top-down approach.

The studio culture informs the life experiences of the architectural design student and is consistent with their temperament. Boyer and Mitgang recognized the student experience, "For much of this century, design has dominated the architecture curriculum at nearly all schools. It is a place - the design studio - where students spend as much as $90 \%$ of their time and energy."[13] Some teachers take into account the personality factors of their students. ${ }^{[14]}$ Keirsey applied Isabel Myers' words when assigning the architects as introverted intuitive tough-minded probing (INTP). ${ }^{[15]}$ The architect temperament described within learning is immersive, "Once caught up in a thought process, that process seems to have a will of its own, and they persevere until they comprehend the issue in all its complexity."[16] An architect may perceive an individual statics problem, and even topic, as mundane and they continue to search for broad meaning. Students circulate in what they perceive as minutiae, entrapped in an interfering programmatic loop.

The parti pris is a reverse content sequence pedagogy beginning with the central big idea symmetrical with the architecture student schemata. "The concept of the parti is used to simplify our understanding of formal organizations. Deriving from the French word partir (to depart), a parti is a point of departure for an architectural idea. In the École des Beaux Arts, the French School of Arts, the term referred to organizational ideas used in quick sketches, or esquisses, which the students prepared in a few hours, and then elaborated for the next few months."[16]

\section{The Parti Pris Course}

The parti pris pedagogy appears in our introductory architectural structures course. The course content sequence parallels the architectural design process by commencing with the broad concepts and ending with refined concepts. The content begins with overall building behavior in response to lateral forces, contracts to framing layouts in response to gravity forces, and concludes with the convergence to rigid body statics and the mechanics of deformable bodies. The process conceptually investigates the external environment of the structural elements prior to their internal behaviors. The general course information, the topical content, lesson plans and instructional strategy, and the assessment protocols describe the parti pris course.

\section{General Course Information}

The course description, content, and requirements respond to the structural systems accreditation criterion. The original course description reads, "Investigation of the basic principles of structural systems through the analysis of overall structural behavior with specific attention to statics and system modeling." $[17]$ The forthcoming catalog description will read, "Introduction to the holistic structural behavior of buildings responding to gravity and lateral forces, and to the individual structural behavior of beams and columns using the principles of rigid body statics and deformable body mechanics." The course is required and accounts for three-credits. The enrollment is about fifteen students in two sections offered annually in the second year of an architecture degree-granting program. The prerequisite courses are Mathematics and Inquiry, Principles of Physics, and Introduction to Building Systems. The textbook is Simplified Engineering for Architects and Builders, which is endorsed by the NCARB. ${ }^{[18,19]}$ 


\section{Topical Content}

The content includes three modules: Verticality - Building Behavior due to Lateral Forces (referred to as verticality); Horizontality - Framing Behavior due to Gravity Loads (horizontality); and Statics and Mechanics - Behavior of Rigid and Deformable Bodies (statics and mechanics). The parti pris sequence is verticality, horizontality, then statics and mechanics (Figure 2). The traditional sequence is statics and mechanics, horizontality, then verticality.

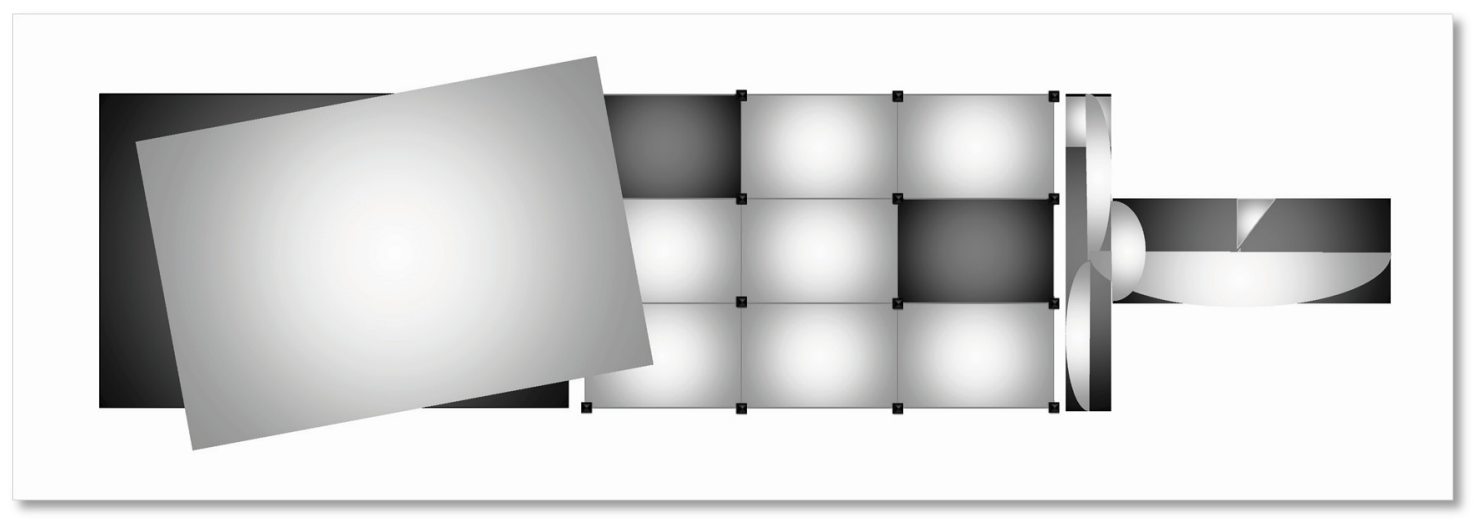

Figure 2. Parti pris.

The verticality module covers the broad building behavior such as drift, overturning, and torsion from lateral forces then constricts to member connectivity. The verticality content includes lectures on lateral loads (wind pressure and earthquake base shear), building behavior (drift, overturning, and torsion), horizontal distribution of story forces (centers of force and stiffness, rigid and flexible diaphragms, and direct and torsional shear), and structural connective philosophies (fully restrained and partially restrained connections). Quantitative theory, intuitive approaches, and case studies support the content. Achievement is based on twenty learning objectives and twenty homework and examination questions associated with specific concepts: lateral forces, wind pressure, earthquake base shear, building overturning, structural irregularities, stabilization techniques for low-rise buildings, stabilization techniques for highrise buildings, frame types, frame characteristics, frame integration, center of force, center of stiffness, building drift, building torsion, frame resistance directionality, direct shear, torsional shear, diaphragm types, connection types, and connectivity and stabilization.

The horizontality module describes the broad behavior of the structural framing due to the gravity forces then narrows to beam depth-to-span relationships. The horizontality content includes lectures on gravity loads (dead, live and patterned live, and load combinations), beams and columns (framing bays and simple behavior), structural layouts (post-and-beam, bearing wall, and additive and subtractive forms), and preliminary sizing of beams, columns, and decking (depth-to-span ratios and tributary areas techniques). Quantitative theory, intuitive approaches, and case studies support the content. Achievement is based on a structural framing graphic vignette of comparable to size and character of the NCARB Practice Program Structural Layout Vignette. The students develop a horizontal solution for a multi-level flat roof with clerestory windows that resists gravity loads. 
The statics and mechanics module characterizes the internal member shears and moments from applied loads then constricts to their properties and stresses. Rigid body statics investigates the internal forces and actions of members through shear, moment, and the deflection of prismatic members, while deformable body mechanics addresses the concepts of first moment and second moments of cross-sectional areas, section modulus, etc. The statics and mechanics content includes lectures on beam types (simply supported, overhanging, cantilever, cable, fixed, and continuous), connections (roller, pinned, and moment), beam and frame reactions, shear and moments in beams, axial forces in planar trusses (method of sections and method of joints), and mechanics of deformable bodies (radius of gyration, moment of inertia, and bending and axial stresses). Achievement is based on twenty learning objectives and twenty homework and exam questions regarding: equations of equilibrium, moment of a force, equilibrium of a particle, equilibrium of a rigid body, material connectivity, degrees of freedom, beam types, failure types, failure locations, loaded diagrams for simple beams, shear diagrams, moment diagrams, deflection diagrams, shear moment and deflection connectivity interdependencies, method of joints, method of sections, moment of inertia, radius of gyration, axial stress, and bending stress.

\section{Lesson Plans and Instructional Strategy}

The lesson plans follow the parti pris inside the individual lectures by introducing the big idea prior to the finer detail. Figure 3 illustrates the sample lecture notes on connectivity. The parti pris lecture begins with the notion of rigid frame buildings (Figure 3(a)) and concludes with shear and moment connective behavior (Figure 3(b)), whereas the traditional sequence formerly began with the member behavior in advance of the broader building stabilization concepts.

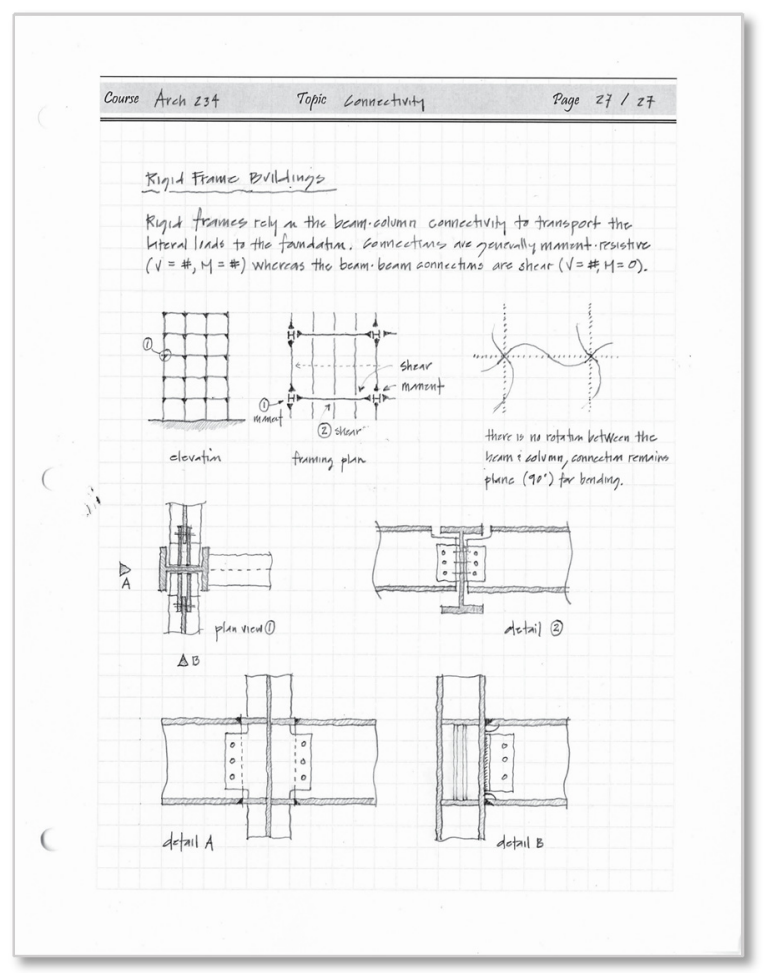

(a)

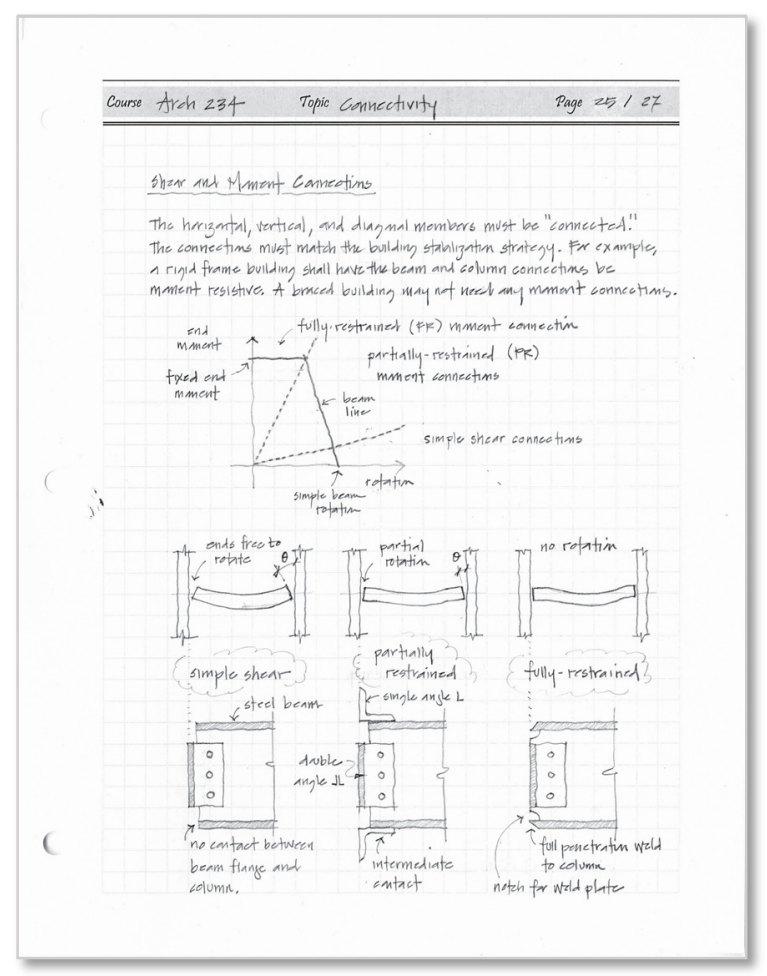

(b)

Figure 3. Lecture notes on connectivity: (a) broad; and (b) refined. 
The instructional strategy is an instructor-centered (teacher-directed) lecture methodology. ${ }^{[20]}$ The lecture model infuses the learning objectives and addresses factual knowledge, fundamental principles, and application. The whiteboard $(7 / 8)$ and PowerPoint $(1 / 8)$ are the primary content dissemination mechanisms. PowerPoint has received harsh criticism from educational scholars as the instructional strategy may launch Death by PowerPoint and PowerPoint Hell anecdotes. ${ }^{\text {[21] }}$ The whiteboard facilitates learning by slowing the instructional pace, engaging the students, permitting longer gestation, and adding retention.

\section{Assessment Protocols}

The student outcomes are part of a holistic approach built to help students learn. ${ }^{[22]}$ A learning cycle balances group and individual activities (Figure 4). The students form into teams to collaborate on homework problem sets and exam preparation. Collaborative activities are part of the learning process. In order to optimize learning, the homework problems have more difficult open-ended questions, while the practice exam problems have closed-ended multiple-choice questions. Each learning objective has a homework problem and an examination question. The students engage work in an eight-stage learning cycle: (a) each team meets to discuss the learning objectives, (b) each student solves each problem set independently to the best of their ability, (c) each team meets and discusses each problem until each is fully understood, (d) the team submits one homework problem set where each student is in agreement with each solution, (e) each student completes one practice exam independently (Bean describes the practice materials as an appropriate means of having students learn as part of the assessment process ${ }^{[23]}$, (f) each team meets to review the instructor assessment of each problem set, (g) each team meets to discuss the practice exam until each learning objective is fully understood, and (h) any student that is still having difficulty is encouraged meet with the instructor and repeat the procedure as necessary. The learning cycle applies to each module. The students receive the close-ended practice problems after they submit the homework problems to avoid the premature discovery of a possible answer array.

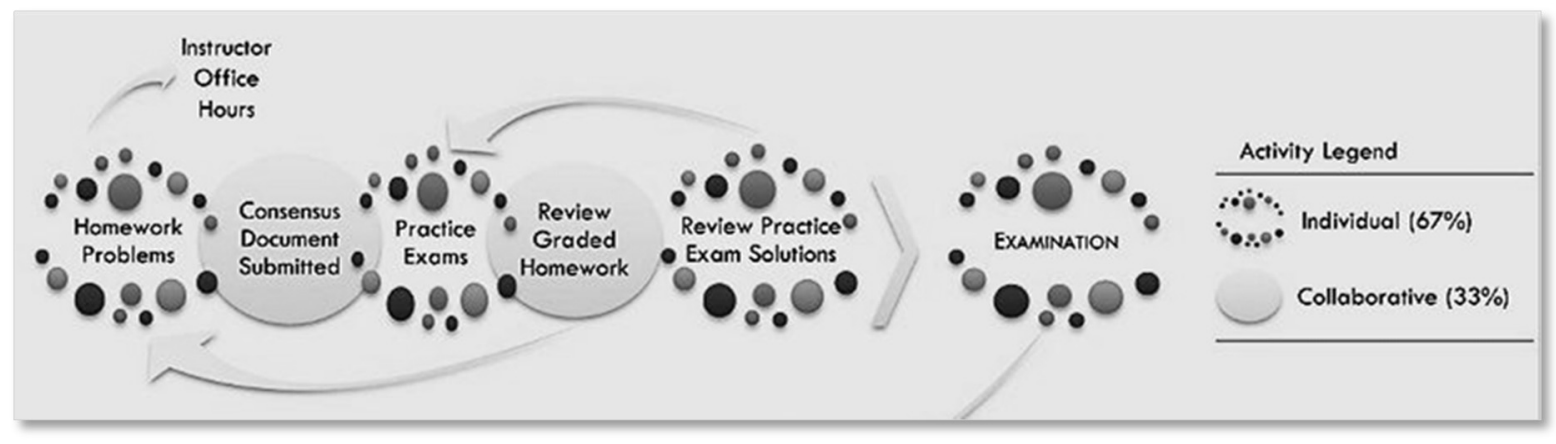

Figure 4. Student work and assessment cycle.

Seven instruments measure student achievement: three group homework packets, three individual examinations, and one individual project. The comparative inquiry relied on three examinations. The distribution gap method in a relative grading system measures the student achievement in the two multiple-choice examinations. ${ }^{[24]}$ The distribution gap method finds the frequency of scores and the opening between the score clusters. The non-graphical exams have 20 multiple-choice questions where $1 / 4$ to $1 / 3$ of the problems are computational based. A partial- 
credit system for the computational problems applies to the correct use and depth of the equations, variables, and subvariables. The competency-based variant method, which is an absolute grading system, measures the graphic exam and project. ${ }^{[24]}$ The method measures the amount of knowledge on an A-F scale for each objective when the student outcomes are primarily visual in nature.

\section{$\underline{\text { Methodology }}$}

The study was borne through the natural evolution and annual adjustments to the architectural structures sequence. The traditional content was not eliciting optimal structures outcomes and inhibiting studio crosspollination. As a result, the introductory course incrementally infused the advanced content over a period of several years where the big idea eventually preceded statics and mechanics. One semester, the two trajectories of exploring the content sequence intersected. In the group comparison tradition, a quantitative analysis explored the unobtrusive data. ${ }^{[25]}$ The null hypothesis is that there is no difference between the traditional group and the parti pris group in terms of performance for architectural structures students. Figure 5 illustrates the content sequence protocol for verticality, horizontality, and statics and mechanics. The research site was Drury University, a small liberal arts institution. A convenience sampling formed the groups through the random enrollment of architecture majors into one of two available sections in the introductory structures courses. The continuous dependent variable (ratio measure) was student performance on two non-graphical exams and one graphical exam. The categorical independent variables (nominal measures) were the traditional group and the parti pris group. The performance instruments were the individual student examinations used to measure academic achievement. The unobtrusive data were two multiple-choice exams and one graphic vignette exam. Reliability was threefold by using similar pilot questions and vignettes from earlier courses to remove ambiguity, by following identical administrative procedures (content, instruction, and assessment) for each module, and avoiding misunderstandings by being available to answer student questions during the test. Construct validity was addressed by observing the expected performance of a structural axonometric project. Inferential statistics methods using the parametric two-tailed $t$-test analyzed the data. ${ }^{[26]}$ The null hypothesis is rejected when the probability value is equal to or less than the significance level of .05.

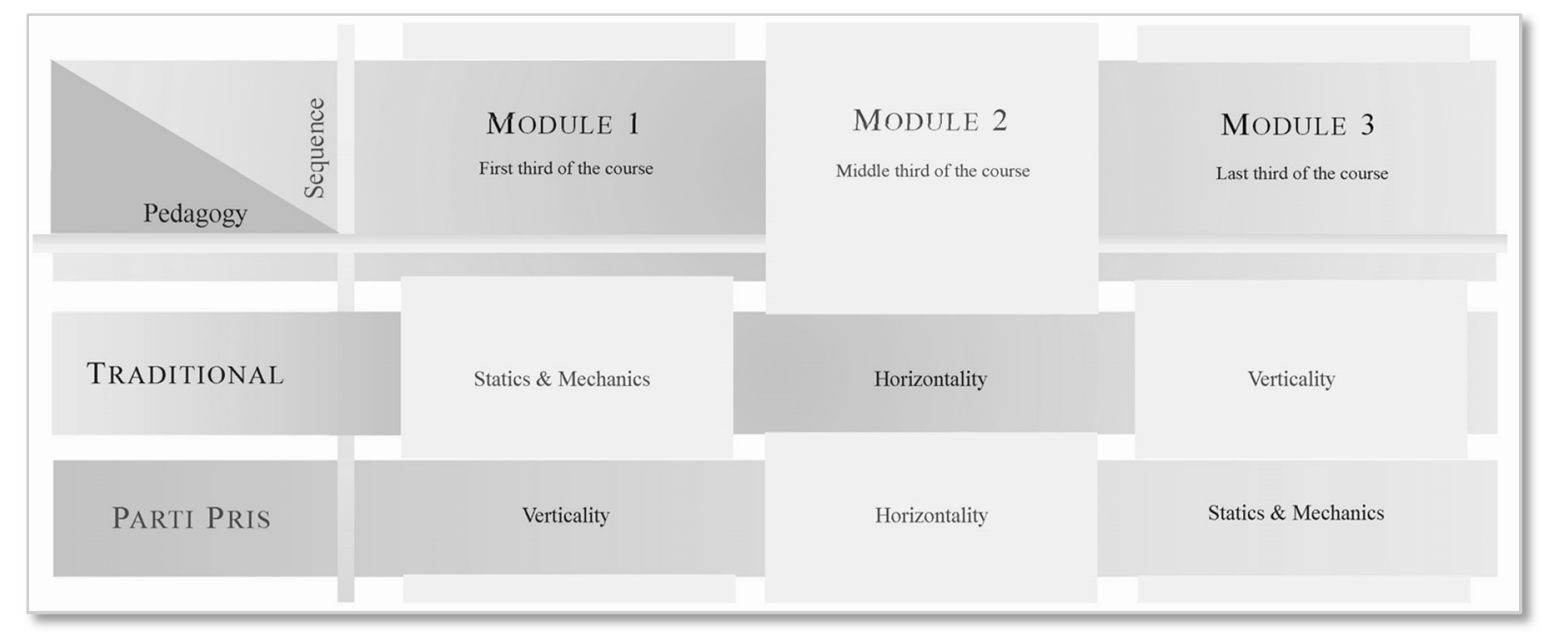

Figure 5. Student performance matrix. 


\section{$\underline{\text { Findings }}$}

The findings for the parti pris group $(n=18)$ and the traditional group $(n=13)$ are presented sequentially for verticality, horizontality, and statics and mechanics. For the verticality outcome, the parti pris group had a higher mean exam score $(\mathrm{M}=82.9)$ than the traditional group $(\mathrm{M}=$ 79.5) from a 100 point scale. The difference was marginally significant. For the horizontality outcome, the parti pris group had about the same mean exam score $(\mathrm{M}=84.2)$ as the traditional group $(\mathrm{M}=83.8)$. The difference was not significant. For the statics and mechanics outcome, the parti pris group had a higher mean exam score $(\mathrm{M}=81.2)$ than the traditional group $(\mathrm{M}=76.7)$. The difference was marginally significant.

\section{$\underline{\text { Discussion }}$}

The findings indicate that the parti pris pedagogy marginally improves performance on nongraphical multiple-choice examinations. The non-graphical exams assess the content extremities (first [verticality] and last [statics and mechanics] modules). The verticality and statics and mechanics performances reject the null hypothesis. Therefore, a marginal difference between the parti pris and traditional pedagogies exists. The non-graphical and graphical exams yield different results, which reveal a difference regardless of the content type. The central big idea and the narrowed content perform better than the traditional content sequencing. The largest performance difference occurs outside of the architecture student schemata, and the smallest difference inside their schemata. The graphics-based studio culture informs the life experiences of the students. The horizontality examination accepts the null hypothesis, which indicates no performance difference between the parti pris and traditional groups for the graphic examination of the horizontality content area. The graphic exam occurred at the same juncture (middle third of the course) for both the parti pris and traditional groups.

\section{Lessons Learned}

There are several lessons learned in the areas of instruction, content, and outcomes. The first lesson is patience. The journey was a few years in the making. The original inspiration occurred as a result of the structural framing layouts being taught in the advanced structures courses. The curricular position for horizontality was not aligned with studio needs and was incrementally moved down into the introductory structures course. Student satisfaction became optimal as the modular content (such as verticality) from other structures courses repositioned and rested in the introductory course. The traditional sequence naturally transformed into the parti pris sequence. The appropriate content stabilization may vary based on the contexts surrounding the student experiences. The second lesson is consistency. The module examinations should occur on the same day to maintain equitable instruction duration. The course was divided into approximately equal thirds, which demands teaching acuity to concurrently balance the content of the verticality and the statics and mechanics modules to be the same length. In addition, the class notes were carefully scripted in advance to create identical module durations. The third lesson is endurance. Teaching the parti pris and the traditional methods during the same semester takes the comparable energy of teaching two different courses rather than two sections of the same course. The fourth lesson is style. The whiteboard is my preferred method of content delivery due to the methodical pace that allows for increased student engagement and the gestation of knowledge. 
PowerPoint may inhibit the number of mental repetitions in the learning process by reducing note taking. The fifth lesson is satisfaction. The student numerical evaluations were significantly higher for the parti pris section. The university uses the IDEA Diagnostic Form Reports for the student evaluations. The overall raw score ratings for the Excellent Teacher category were 3.8 and 4.5 for the traditional and parti pris groups, respectively, whereas the IDEA adjusted scores were 3.7 and 4.7 from a 5.0 scale. The overall ratings for the Excellent Course category were 3.6 and 4.3 for the traditional and parti pris groups, respectively, while the adjusted scores were 3.4 and 4.7. Student satisfaction alone is strong incentive to consider pedagogical change. The written comments were very scarce. Only one student in the parti pris section had a written comment, "I honestly enjoyed this class a great deal. [The teacher] makes hard subject matter easier and attainable." One student in the traditional section wrote, "The way this class is set up doesn't really make students learn." The sixth lesson is integration. The parti pris group was better prepared for their final visualization project connected to their studio outcomes. The project was an exploded isometric structural diagram of a design studio project to illustrate the relationship between structure and architectural space. Figure 6 shows one student submittal from the parti pris group.

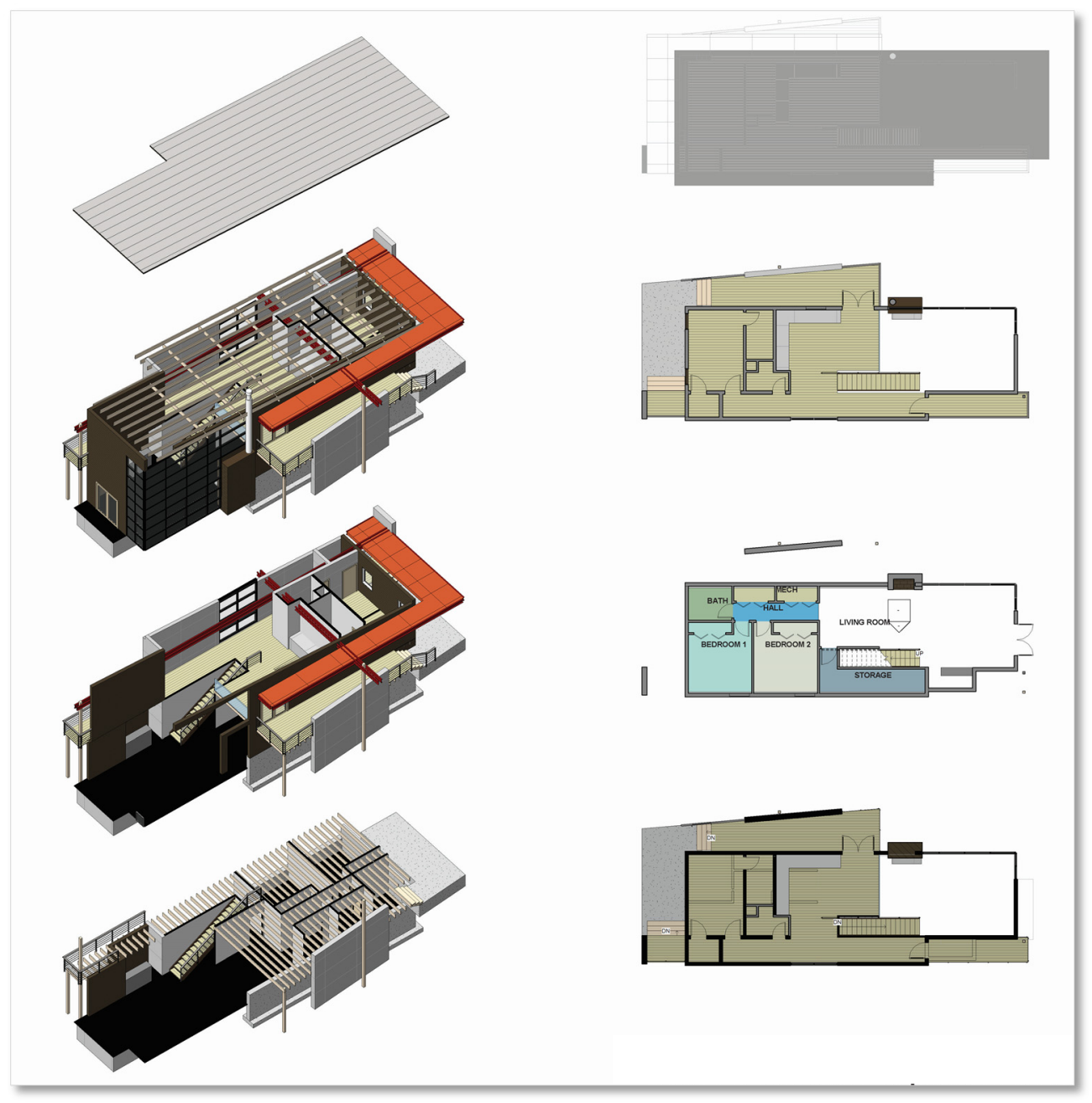

Figure 6. Studio integration project. 


\section{Limitations of the Study}

Two limitations in the study inhibit making broad generalizations about the findings. First, the convenience sampling permitted only a small sample size of thirty-one total students. The limitations do not influence replication, but do inhibit generalizing to larger populations. Second, two potentially intervening variables are student fatigue and guessing that researchers should address. ${ }^{[27]}$ The rigorous demands imposed by the architectural design studios induce fatigue. The studio environment sometimes recognizes the lack of sleep as a badge of honor amongst the students. To minimize fatigue, the exams were not scheduled proximate to major studio project due dates. Multiple choice exams may encourage guessing. Therefore, partial credit was permitted for questions that involved computations.

\section{Comparison with Existing Studies}

Scholars have performed pedagogical trials of reverse content in the lecture and in the studio formats to connect design thinking and structures. Kate Simonen of the California College of Arts and Crafts deployed a reverse sequence in the lecture format. She taught structural design backwards. The motivations were not based on scientific psychology, but rather a fundamental and pragmatic approach founded on the timing needed to connect with studios. Simonen recognized "the primary benefit of teaching structures in this order is that students are able to comfortably gain the ability to select and configure structural systems early enough in their education to utilize this knowledge in their early design studios and develop their skill in integrating structure with architecture." ${ }^{[28]}$ Christine Thorodoropoulos chose a reverse sequence in a studio focusing on tensile structures to enhance the creativity prior to engaging the programmatic constraints. ${ }^{[29]}$ Their work commences with broad notions and ends with refined content that may inhibit design outcomes when introduced earlier. The parti pris sequence is consistent with this approach by engaging the internal member behavior in the last module.

\section{Implications for Future Research}

The future research of parti pris pedagogy should include qualitative studies to capture the nature of the pedagogy and should include quantitative studies to narrow the data and capture the detailed student performance at the concept level. The study brings about a broad awareness that reversing content elicits an improvement to the non-graphical student performance of architecture students in structures courses at a liberal arts institution. Further research should refine the data to align with specific concepts. Architectural structures concept inventories should be developed and builds upon the inventories in a similar manner as the statics concept inventories in engineering programs. ${ }^{[30]}$

\section{Overall Significance}

Pedagogy must foster and not 'interfere' with learning. How we learned structures might not translate into an appropriate pedagogy for other learners at a different institution or in a different degree program. Engineering instructors may be carrying the historical weight of having received an education in a manner appropriate for their needs, but an education that may be incompatible with an architecture audience. The architecture and engineering disciplines will benefit if this paper redirects the discourse from mathematics to a broader discussion of the student mental frameworks in structures pedagogy. 
Bibliography

[1] Beeston, D. (2009). The early years of Albert Einstein: When viewed through the lens of current theory and research were there signs of giftedness? APEX, 15(4), 56-77.

[2] Allen, E. (Ed.). (1992, Winter). Polarities: Teaching structures. Connector, 1(1) 1.

[3] Salvadori, M. (1958). Teaching structures to architects. Journal of Architectural Education, 13(1), 3-8.

[4] Bender, R. (1976, June). The teaching of structural design. Association of Collegiate Schools of Architecture [ACSA Memo]. Reprinted and cited by Black, R. G., \& Duff, S. (1994). A model for teaching structures: Finite element analysis in architecture education. Journal of Architecture Education, 48(1), 38-55.

[5] Faoro, D. F. (1994, Fall). Report of survey on structures curricula. Connector, 3(2), 2.

[6] Bruning, R. H. (1994). The college classroom from the perspective of cognitive psychology. In K. W. Prichard \& R. M. Sawyer (Eds.) Handbook of college teaching: Theory and applications (pp. 3-22). Westport, CN: Greenwood Press.

[7] American Collegiate Schools of Architecture (ACSA). Available at http://www.acsa-arch.org/schools/guideto-architectural-education/overview/architecture-programs (accessed October 15, 2012).

[8] National Architectural Accreditation Board [NAAB]. (2013, August 29). 2014 conditions for accreditation: First draft. Washington, DC: NAAB.

[9] National Council of Architectural Registration Boards [NCARB]. (2012). NCARB by the numbers. Washington, DC: NAAB.

[10] Kamphoefner, H. (1958). Foreword to "Teaching structures to architects." Journal of Architectural Education, 13(1), 3-8.

[11] Littmann, W. (2000). Assault on the école: Student campaigns against the Beaux Arts, 1925-1950. Journal of Architectural Education, 53(3), 159-166.

[12] Anderson, R. C., \& Pichert, J, W. (1978). Recall of previously unrecallable information following a shift in perspective. Journal of Verbal Learning and Verbal Behavior, 17, 1-12.

[13] Boyer, E. L., \& Mitgang, L. D. (1996). Building community: A new future for architectural education and practice. Princeton, NJ: The Carnegie Foundation for the Advancement of Teaching.

[14] Carskadon, T. G. (1994). Student personality factors: Psychological type and the Myers-Briggs Type Indicator. In K. W. Prichard \& R. M. Sawyer (Eds.) Handbook of college teaching: Theory and applications (pp. 69-82). Westport, CN: Greenwood Press.

[15] Keirsey, D. (1998). Please understand me II: Temperament, character, intelligence. Del Mar, CA: Prometheus Nemesis Book Co.

[16] Gargus, J. (1994). Ideas of order: A formal approach to architecture. Dubuque, IA: Kendall/Hunt Publishing Company.

[17] www.drury.edu

[18] Ambrose, J., \& Tripeny, P. (2011). Simplified engineering for architects and builders (11th ed.). New York: John Wiley \& Sons, Inc.

[19] www.ncarb.org

[20] Ekeler, W. J. (1994). The lecture method. In K. W. Prichard \& R. M. Sawyer (Eds.) Handbook of college teaching: Theory and applications (pp. 85-98). Westport, CN: Greenwood Press.

[21] Tufte, E. R. (2003). The cognitive style of PowerPoint. Cheshire, CN: Graphics Press LLC.

[22] Bain, K. (2004). What the best college teachers do. Cambridge, MA: Harvard University Press.

[23] Bean, J. C. (2001). Engaging ideas: The professor's guide to integrated writing, critical thinking, and active learning in the classroom. San Francisco: Jossey-Bass Publishers.

[24] Wright, D. L. (1994). Grading student achievement. In K. W. Prichard \& R. M. Sawyer (Eds.) Handbook of college teaching: Theory and applications (pp. 439-450). Westport, CN: Greenwood Press.

[25] Creswell, J. W. (2005). Educational research: Planning, conducting, and evaluating quantitative and qualitative research. Upper Saddle River, NJ: Pearson Merrill Prentice Hall. 
[26] Gravetter, F. J., \& Wallnau, L. B. (2004). Statistics for the behavioral sciences (6 ${ }^{\text {th }}$ ed.). Belmont, CA: Thomson Wadsworth.

[27] Rudner, L. M. (1993). Test evaluation [online]. Available at www.ericae.net

[28] Simonen, K. (2002, Spring-Fall). We've been teaching structural design backwards. Connector, 11(1/2), 1-2.

[29] Thorodoropoulos, C. (2001, Spring). Designing backwards. Connector, 10(1), 12.

[30] Steif, P. S., \& Dantzler, J. A. (2005). A statics concept inventory: Development and psychometric analysis. Journal of Engineering Education, 94(4), 363-371. 